\title{
A Monolithic Double-balanced Upconverter for millimeter-wave Point-to-Multipoint Distribution Systems
}

\author{
K. S. Ang and I. D. Robertson \\ Microwave and Systems Research Group \\ School of Electronic Engineering, Information Technology and Mathematics \\ University of Surrey \\ Guildford, Surrey GU2 7XH, UK \\ k.ang@eim.surrey.ac.uk
}

\begin{abstract}
The design and performance of a monolithic double-balanced upconverter for mm-wave point-tomultipoint distribution systems are presented. Individual building blocks including the LO / RF balun, IF balun, LO / RF power amplifier, and the doublebalanced mixer are also fabricated and tested. The complete upconverter, measuring $3 \times 3.2 \mathrm{~mm}$, upconverts the 3 - $5 \mathrm{GHz}$ IF to $40.5-43.5 \mathrm{GHz}$ band with $20 \mathrm{dBm}$ output power.
\end{abstract}

\section{INTRODUCTION}

Double-balanced mixers (DBMs) provide inherent port isolations, LO AM noise rejection, even-order mixing product suppression and high dynamic range. These desirable mixer properties are often exploited to realise high performance receivers with low intermodulation distortion [1-10]. These properties, however, are equally desirable in transmitter applications for upconverting signals with low spurious output. In this paper, the DBM is employed to develop a high power level upconverter for point-to-multipoint distribution systems.

\section{CIRCUIT STRUCTURES}

\section{A. Double-Balanced Mixer}

Fig. 1 shows the building blocks of a DBM. It consists of an arrangement of four mixing elements with LO, RF and IF baluns. Schottky diodes have been the primary device employed for the mixing elements [1-4]. Due to their highly non-linear characteristics, they inherently exhibit poor intermodulation and spurious response properties. Moreover, in the monolithic realm, they are usually implemented using some derivative of the MESFET structure. This often results in suboptimum diodes with inferior mixer performance in comparison with mixers using discrete diodes.

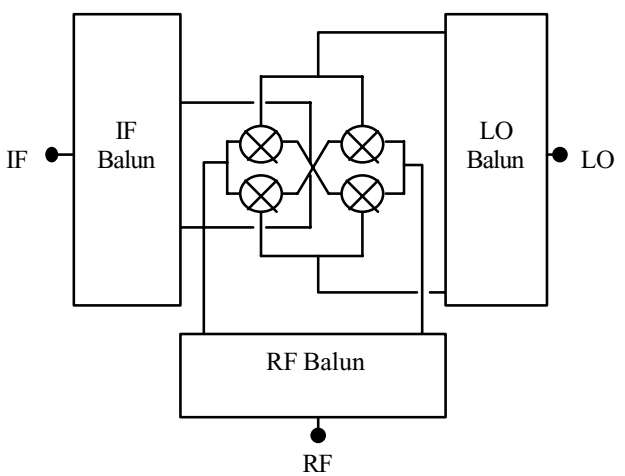

Fig. 1: Double-balanced mixer block diagram

Dual-gate FETs, which provide separate gates for the LO and RF have also been used to realise monolithic DBMs [10]. However, dual-gate FET mixer designs usually require accurate non-linear models, which are often highly complex and not readily available. They are also subject to instability problems.

Resistive FET mixers, which exhibit inherently low intermodulation properties have recently been employed in DBMs [5-9]. Other advantages of the resistive FET mixer include low DC power consumption and guaranteed stability. Monolithic resistive DBMs operating up to $\mathrm{Ku}$-band have been reported $[6,8]$. The mm-wave resistive DBM presented here employs 0.25 $\mu \mathrm{m}$ AlGaAs/InGaAs PHEMTs.

Besides the mixing elements, the other aspect of DBM design is the LO, RF and IF baluns. Many previous monolithic designs have employed active baluns for the LO and lumped element baluns for the low frequency IF. Although active baluns reduce the LO power requirements by offering gain, they are often sensitive to the operating conditions, require several DC bias connections and have poor noise and linearity performance. In the design presented here, an alternative approach using a passive LO balun in conjunction with an amplifier is employed. 


\section{B. Upconverter}

Fig. 2 shows the block diagram and primary specifications of the upconverter. A single power amplifier is designed to cover both the $\mathrm{LO}$ and $\mathrm{RF}$ bands. Instead of lumped element components, the IF balun employs a dual spiral transformer. This results in a more robust and compact design with low component count.

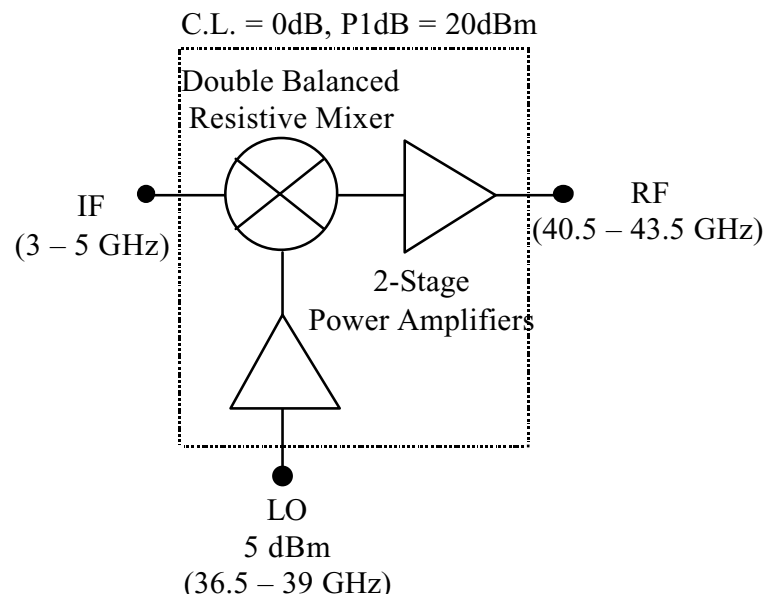

Fig. 2: Upconverter block diagram

\section{MMIC REALISATIONS AND PERFORMANCES}

The development of the upconverter adopts a modular approach in which individual building blocks are fabricated and tested to ensure first-pass success. The fabricated circuits include the LO / RF balun, IF balun, DBM, LO / RF power amplifier, and the complete upconverter. The MMICs are fabricated by Marconi Caswell Ltd. using the standard H40 foundry process which employs $0.25 \mu \mathrm{m}$ gate length $\mathrm{AlGaAs} / \mathrm{InGaAs}$ PHEMTs on a $100 \mu \mathrm{m}$ thick GaAs substrate.

\section{A. LO / RF Balun}

The LO / RF balun is realised by combining two interdigital Lange couplers, as shown in Fig. 3. To realise the tight coupling for broadband operation and matched odd- and even- mode phase velocities, narrow line widths and gaps are required. However, compromises have to be made to stay within the foundry process limitations for reasonable yield. The widths and gaps of the interdigital fingers are designed to be $9 \mu \mathrm{m}$ and $6 \mu \mathrm{m}$ respectively. The lengths of the couplers are $700 \mu \mathrm{m}$ for operation centered at $38 \mathrm{GHz}$.

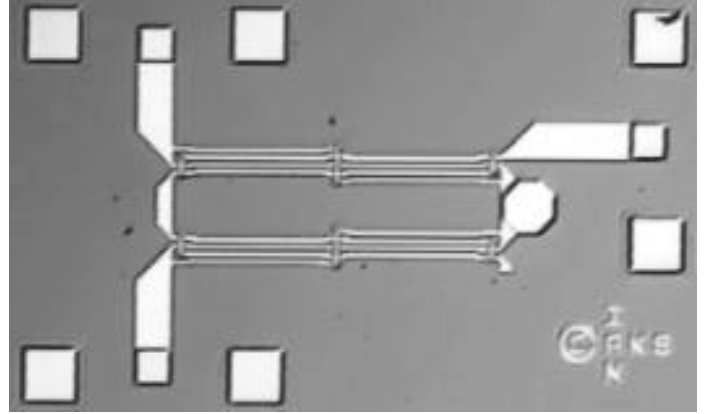

Fig. 3: LO / RF balun MMIC ( $0.8 \times 1.4 \mathrm{~mm})$

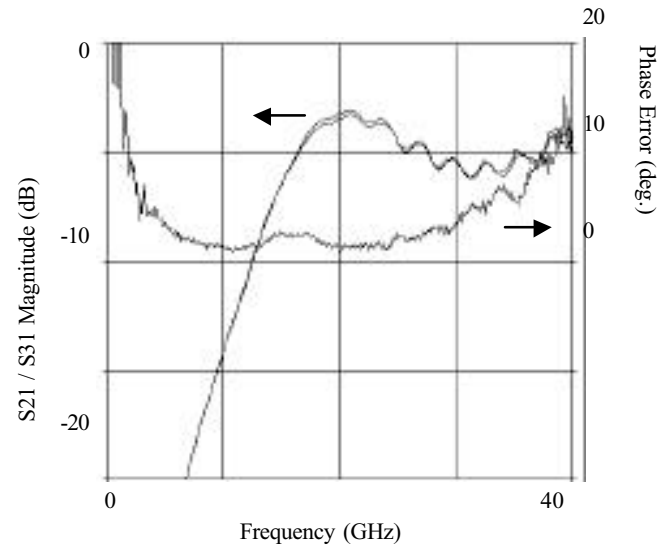

Fig. 4: LO / RF balun performance

Fig. 4 shows the performance of the LO / RF balun. Both the balun $S 21$ and $S 31$ exhibit an average value of $4.5 \mathrm{~dB}$ with a ripple of $\pm 1.5 \mathrm{~dB}$. Despite the ripples in the performance, the amplitude and phase imbalances between the two outputs are less than $0.5 \mathrm{~dB}$ and $10^{\circ}$ over the 20 to $40 \mathrm{GHz}$ range.

\section{B. IF Balun}

For the lower frequency IF balun, a pair of spiral transformers is used in placed of the interdigital couplers. Fig. 5 shows a photograph of the fabricated MMIC which consists of two oppositely wound twincoil transformers connected in series. The input coil is terminated with an open-circuit while the output coils are terminated in a short-circuit. Each twin-coil transformer operates as a quadrature coupler with a center frequency roughly corresponding to an unwound length of a quarter wavelength. In the present design, the operating bandwidth is designed to be between 3 to $5 \mathrm{GHz}$. The spiral transformers have 4.5 turns with track width and gap of $12 \mu \mathrm{m}$ and $8 \mu \mathrm{m}$, respectively. These dimensions are well within the fabrication limits of the foundry. 


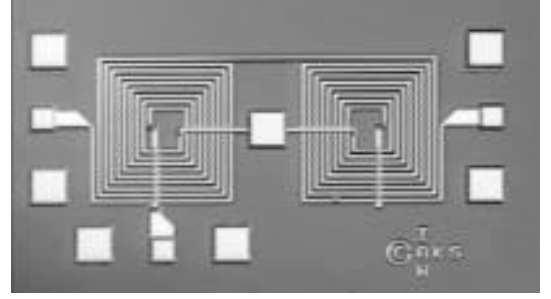

Fig. 5: IF balun MMIC $(0.7 \times 1.5 \mathrm{~mm})$

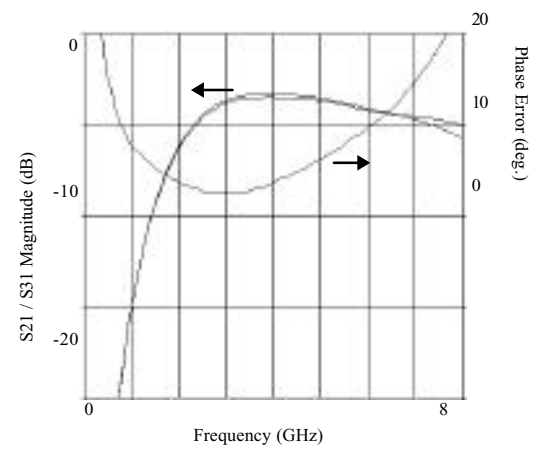

Fig. 6 IF balun performance

As shown in Fig. 6, the $S 21$ and $S 31$ of the IF balun tracks each other closely up to $6 \mathrm{GHz}$. The insertion loss at the centre frequency is less than $0.5 \mathrm{~dB}$ at mid-band. The amplitude and phase imbalances are respectively within $0.2 \mathrm{~dB}$ and $10^{\circ}$ from 2 to $6 \mathrm{GHz}$.

\section{Double-balanced Mixer}

Fig. 7 shows the photograph of the resistive DBM, consisting of four PHEMTs, LO/RF and IF baluns. Reverse gate bias for the PHEMTs are applied to the LO balun through a mesa resistor. Particular attention was devoted to the layout of the circuit in order to maintain symmetry and to minimise transmission line crossings in the circuit; these introduce parasitics and may degrade the performance of the mixer. Most of the cross-overs and discontinuities were placed in the IF path as the IF is low enough that these are acceptable.

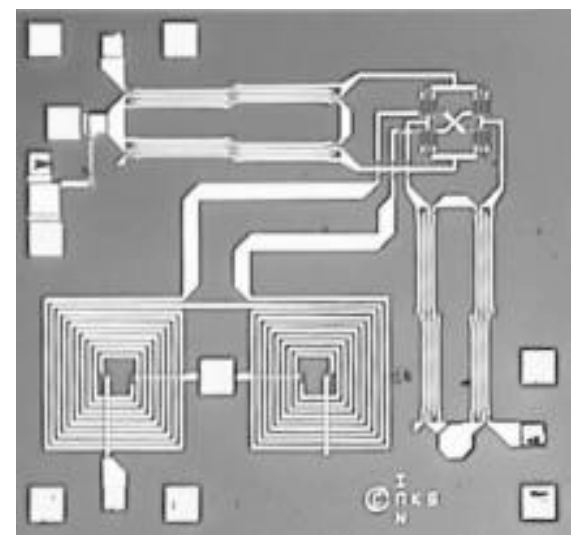

Fig. 7: Double-balanced mixer MMIC (1.6 x 1.7mm)

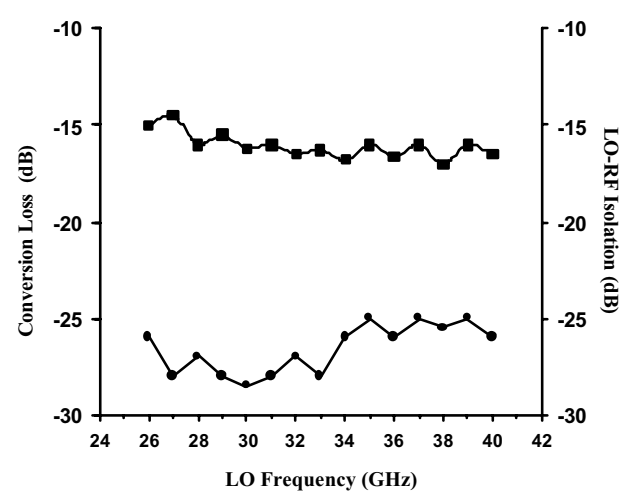

Fig. 8 Double-balanced resistive mixer performance

Having verified the operating frequency ranges and performances of the LO / RF and IF baluns, the upconverter performance of the double-balanced mixer was measured. A fixed IF of $4 \mathrm{GHz}$ at $0 \mathrm{dBm}$ was applied to the mixer, while the LO was swept from 26 to $40 \mathrm{GHz}$. Across the frequency band, a conversion loss of $16 \pm 1$ $\mathrm{dB}$ was obtained with over $25 \mathrm{~dB}$ of LO-RF isolation, as shown in Fig. 8. The high conversion loss was due to the insufficient LO power level applied, which was $10 \mathrm{dBm}$. Unfortunately, this was the maximum LO power available for the measurement. With $10 \mathrm{dBm}$ LO power, an input IP3 of $18 \mathrm{dBm}$ was obtained from two-tone intermodulation measurements with $0 \mathrm{dBm}$ IF power.

\section{LO / RF Power Amplifier}

Fig. 9 shows a photograph of the LO/RF power amplifier. It is a two-stage power amplifier employing three 4x60 PHEMTs. The output matching network transforms the $50 \Omega$ load to the optimal load impedances of the output stage. The interstage and input matching networks provide an overall flat gain response with good input return loss over the required bandwidth. These design objectives were achieved in the fabricated MMIC, as shown in the measured results of Figs. 10 and 11.

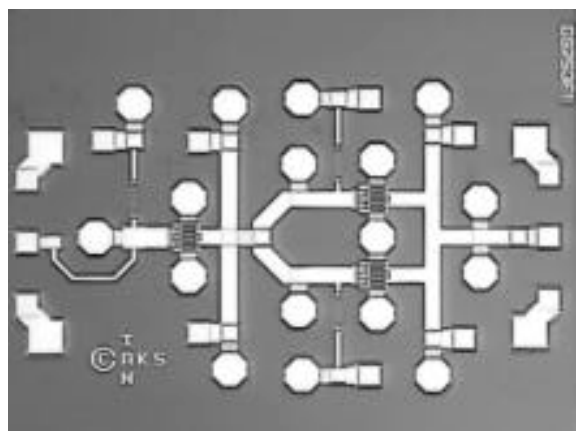

Fig. 9: LO/RF power amplifier MMIC (1 x1.7 mm) 


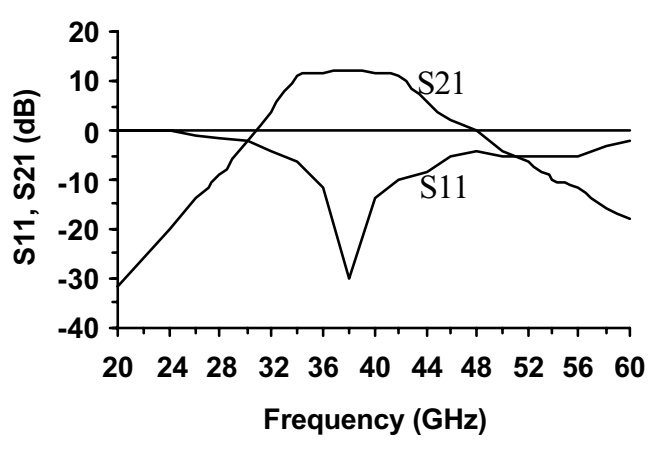

Fig. $10 \mathrm{LO} / \mathrm{RF}$ power amplifier small-signal performance

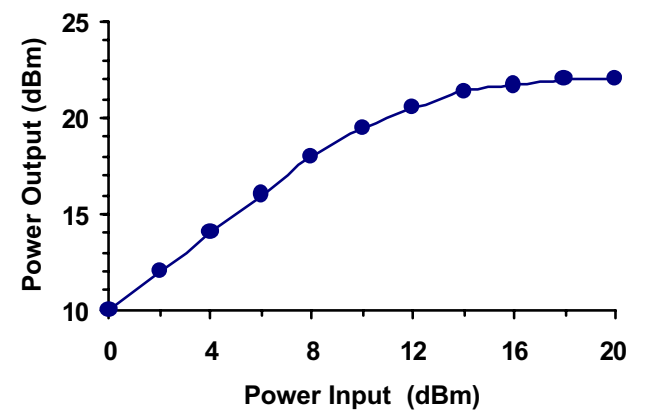

Fig. 11: LO/RF power amplifier output power

performance

Fig. 10 shows a flat gain response of $10 \mathrm{~dB}$ from 33.5 to $43.5 \mathrm{GHz}$ with better than $10 \mathrm{~dB}$ input return loss. The output P1dB obtained from Fig. 11 is better than $20 \mathrm{dBm}$.

\section{E. Complete Upconverter}

By incorporating all the building blocks together on a single MMIC, the complete upconverter shown in Fig. 12 , measuring $3 \times 3.2 \mathrm{~mm}$, is realised. With the addition of the LO and RF power amplifiers, the upconverter achieved $0 \mathrm{~dB}$ conversion loss with $5 \mathrm{dBm}$ LO input power.

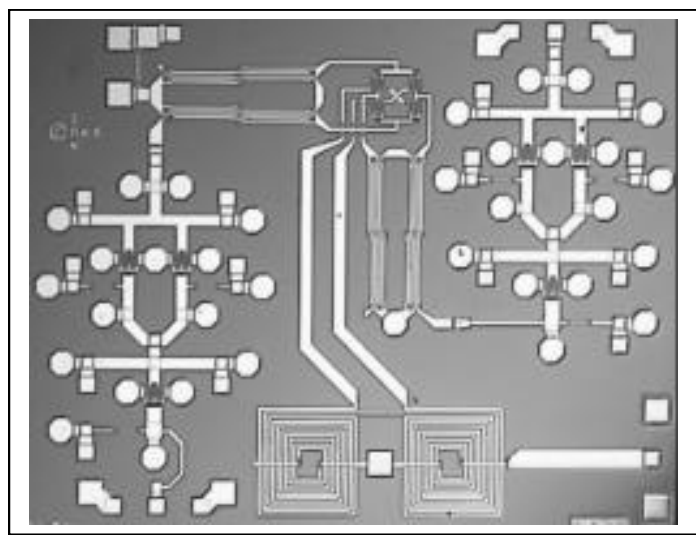

Fig. 12: Complete upconverter MMIC (3 x $3.2 \mathrm{~mm})$

\section{CONCLUSIONS}

A millimeter-wave double-balanced upconverter has been developed. Resistive PHEMTs, which exhibit low intermodulation are employed as the mixing elements. The LO/RF and IF baluns utilise compact passive coupling structures with good amplitude and phase balance, which are critical to the mixer performance. Power amplifiers are included for the LO and RF ports to achieve low input LO input drive and high output power requirements. The performances of the various building blocks have been individually verified to ensure the successful application of the upconverter for millimeterwave point-to-multi-point distribution systems.

\section{REFERENCES}

[1] A. M. Pavio, et. al. "Double balanced mixers using active and passive techniques," IEEE Trans. Microwave Theory Tech., vol. MTT-12, pp. 19481956, 1988 .

[2] T. H. Chen, et. al., "Broadband monolithic passive baluns and monolithic double-balanced mixer," IEEE Trans. Microwave Theory Tech., vol. MTT12, pp. 1980-1986, 1991.

[3] S. A. Maas and K. W Chang, "A broadband, planar, doubly balanced monolithic Ka-band diode mixer," IEEE Trans. Microwave Theory Tech., vol. MTT-41, pp. 2330-2335, 1993.

[4] S. A. Maas, et. al., "An 18-40 GHz monolithic ring mixer," in 1998 IEEE Radio Frequency Integrated Circuits Symp. Dig. pp. 29-32.

[5] S. Weiner, D. Neuf and S. Spohrer, "2-18 GHz double balanced MESFET mixer with $+30 \mathrm{dBm} 3^{\text {d }}$ order intercept," in 1988 MTT-S Int. Microwave Symp. Dig., 1097-1100.

[6] T. H. Chen, et. al. "A double balanced 3-18 GHz resistive HEMT monolithic mixer," in 1992 MTT-S Int. Microwave Symp. Dig., pp. 459-462.

[7] F. D. Flaviis and S. A. Maas, "X-band doubly balanced resistive FET mixer with very low intermodulation," IEEE Trans. Microwave Theory Tech., vol. MTT-43, pp. 457-460, 1995.

[8] M. C. Tsai, et. al., "A compact wideband balanced mixer," in 1994 IEEE Microwave and MillimeterWave Monolithic Circuits Symp. Dig., pp. 135 138.

[9] JF. Villemazet, et. al. "New compact double balanced monolithic down-converter application to a single chip MMIC receiver for satellite equipment," in 1998 MTT-S Int. Microwave Symp. Dig., pp. 853-856.

[10] D. Neilson, et al., "A broadband upconverter IC," in 1992 MTT-S Int. Microwave Symp. Dig., pp. 455458 